\title{
MicroRNAs in CSF_biomarkers of brain cancers and disease activity
}

MicroRNAs (miRNAs) in cerebrospinal fluid (CSF) are markers of brain cancer that can be used to distinguish between different types of cancers and may reflect disease activity, according to results from a new study, published in Neuro-oncology.

Many biological processes are regulated by miRNAs, and these molecules are known to be dysregulated in cancer. "Studies suggest that practically every cancer investigated has a distinct miRNA signature," says Anna Krichevsky, senior author of the study. The researchers investigated miRNAs in the CSF of 19 patients with glioblastoma multiforme (GBM), 74 patients with metastatic cancers, and 15 non-neoplastic controls.

Levels of miR-10b and miR-21 were highly elevated only in patients with GBM or brain metastases, suggesting these miRNAs are markers of primary and metastatic brain cancer. Members of the miR-200 family were found solely in CSF of patients with brain metastases, and could be used to discriminate patients with metastatic brain cancer from those with primary brain cancers. Combined analysis of seven miRNAs enabled discrimination between GBM and metastatic brain cancers with 95\% accuracy. CSF levels of miRNAs also correlated with disease activity: miR-10b, miR-21 and miR-200 levels were lower in patients with GBM or brain metastases who were in remission than in those who had active disease.

"We still need to analyse more specimens and additional pathological conditions to develop miRNA biomarkers into clinically useful diagnostic and disease-monitoring tools, but this new approach looks very promising," concludes Krichevsky.

\section{Katy Malpass}

Original article Teplyuk, N. M. et al. MicroRNAs in cerebrospinal fluid identify glioblastoma and metastatic brain cancers and reflect disease activity. Neuro Oncol. doi:10.1093/neuonc/nos074 